\title{
Peripheral Sensory Neuropathy, CTCAE
}

National Cancer Institute

\section{Source}

National Cancer Institute. Peripheral Sensory Neuropathy, CT CAE. NCI Thesaurus. Code C143752.

A disorder characterized by damage or dysfunction of the peripheral sensory nerves. 